\title{
Zum Thermotransport in Wolfram
}

\author{
G. M. Neumann \\ Osram-Studiengesellschaft, Augsburg \\ (Z. Naturforschg. 22 a, 393-395 [1967] ; eingegangen am 14. Oktober 1966)
}

\begin{abstract}
Aus der an hochgeglühten Wolframdrähten im elektrischen Potentialgradienten und im Temperaturgradienten auftretenden Oberflächenstrukturierung ergibt sich im Zusammenhang mit der elektrischen Uberführung von Wolfram zur Kathode ein Thermotransport von Wolfram zum kälteren Probenteil. Die Transportwärme wird an Hand der Stufenstruktur sowie aus theoretischen Daten abgeschätzt.
\end{abstract}

In metallischen Festkörpern herrscht bei höherer Temperatur stets ein gewisses Fehlordnungsgleichgewicht, das den Platzwechsel einzelner Gitterbausteine ermöglicht. Der zunächst ungeordnete Platzwechsel erhält bei Vorhandensein einer gerichteten Kraft eine bevorzugte Bewegungskomponente in Richtung des treibenden Potentialgefälles. Dabei lassen sich je nach der Art der treibenden Kraft drei Fälle unterscheiden: die isotherme Diffusion unter dem Einfluß eines chemischen Potentialgradienten, der Elektrotransport unter dem Einfluß eines elektrischen Potentialgradienten und der Thermotransport unter dem Einfluß eines Temperaturgradienten.

Die Auswirkung einer gerichteten Kraft tritt sehr anschaulich beim "Gleichstromeffekt" ${ }^{1}$ in Erscheinung. Dieser Effekt äußert sich beim Glühen von Drähten und Bändern der hochschmelzenden kubischraumzentrierten Übergangsmetalle in einem Gleichstromfeld oder einem Temperaturgradienten durch die Ausbildung einer eigenartigen flächen-spezifischen, stufenförmigen Oberflächenstruktur (Abb. 1), die beim Glühen im Wechselstromfeld ausbleibt. Eine Reihe von Autoren ${ }^{2-6}$ hat festgestellt, daß die Orientierung der Gleichstromstruktur von der Richtung des elektrischen Feldes abhängig ist. Sie läßt sich daher als Indikator für die Richtung der Wanderung der am Platzwechsel beteiligten Gitterbausteine heranziehen. Die Untersuchungen zum Gleichstromeffekt an Wolfram haben dabei ergeben, daß die über die (110)-Fläche der Drähte zusammenwachsende „Fischgrätenstruktur“ mit ihrer Pfeilspitze stets zur Kathode gerichtet ist. Unabhängig davon haben Untersuchungen des Elektrotransportes von Wolfram mit Hilfe einer radioaktiven SandwichMethode $^{7}$ eine Überführung des Wolframs zur Kathode ergeben. „Fischgrätenstruktur" und Materiefluß stimmen also in ihrer Richtung überein. Damit ist aber auch ein ausgezeichnetes Charakteristikum der Transportrichtung der Gitterbausteine gegeben. Untersucht man nun an Hand dieser Betrachtungen die im Gebiet eines Temperaturgradienten auftretende Oberflächenstrukturierung eines Wolframdrah-

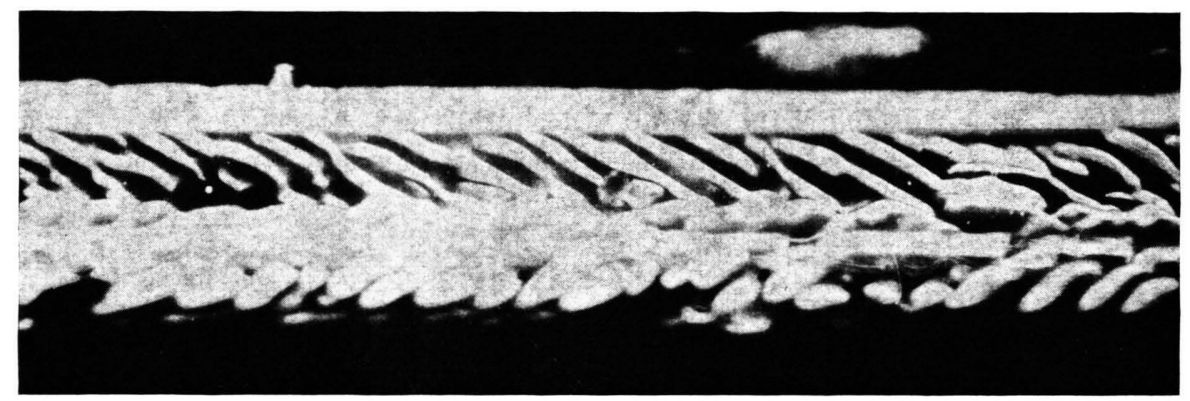

Abb. 1. „Fischgrätenstruktur“ beim Gleichstromeffekt an Wolframdrähten. Kathode rechts.

1 Zusammenfassende Arbeit: G. M. Neumann, W. Hirschwald u. I. N. Stranski, Z. Metallk. 57, 836 [1966].

2 R. P. Johnson, Phys. Rev. 54, 459 [1938].

3 D. B. Langmuir, Acta Met. 5, 13 [1957].

4 E. Heinrich, Dissertation, Technische Universität Berlin 1953.
5 K. U. Kehrer, Diplomarbeit, Universität Tübingen 1954.

${ }^{6}$ G. M. Neumann, Dissertation, Technische Universität Berlin 1965.

7 G. M. Neumann u. W. Hirschwald, Z. Naturforschg. 22 a, 388 [1967]. 
tes, so zeigt sich, daß die Pfeilspitze der „Fischgrätenstruktur" zum kälteren Probenende hinweist. Daraus folgt, daß Wolfram unter dem Einfluß eines Temperaturgradienten zum kälteren Teil transportiert wird und daß die Transportwärme $Q^{*}$ einen positiven Wert besitzt.

Eine direkte experimentelle Bestimmung des Thermotransportes an Wolfram ist mit Hilfe von Oberflächenmarkierungen infolge der auftretenden Oberflächenstrukturierung überhaupt nicht und mit Hilfe von inerten Fremdkörpern wegen der Temperaturunbeständigkeit der verwendeten Markierungen nur äußerst schwierig durchführbar. So bleiben als Möglichkeiten für die Bestimmung der Transportwärme der Thermodiffusion von Wolfram die Abschätzung aus der im Temperaturgradienten auftretenden Stufenstruktur und die Berechnung an Hand theoretischer Úberlegungen.

Für den Materiestrom in einem Temperaturgradienten gilt allgemein die Beziehung

$$
I=\frac{D}{f R T^{2}} Q^{*} \frac{\mathrm{d} T}{\mathrm{~d} x}
$$

mit $I=$ Materiestrom, $D=$ Selbstdiffusionskoeffizient, $f=$ Korrelationsfaktor (krz.-Gitter: 0,727 ), $R=$ Gaskonstante, $T=$ Temperatur, $Q^{*}=$ Transportwärme und $\mathrm{d} T / \mathrm{d} x=$ Temperaturgradient. Betrachtet man das Volumen der Oberflächenstufen als Maß für den Materiestrom, so kann man über den Zusammenhang

$$
V=I t / d
$$

mit $V=$ Stufenvolumen, $d=$ Stufenabstand und $t=$ Zeit zum Aufbau der Stufe, aus (1) und (2) durch Einsetzen der bekannten Daten die Transportwärme $Q^{*}$ abschätzen.

Mit einem mittleren Stufenabstand bei $T=1800$ ${ }^{\circ} \mathrm{K}$ von $d=5 \cdot 10^{-4} \mathrm{~cm}$, einem Diffusionskoeffizien- ten ${ }^{8} D=1,1 \cdot 10^{-17} \mathrm{~cm}^{2} / \mathrm{s}$ und einer Aufbauzeit von rund 70 Stunden, eine Zeit, nach der die Stufenbildung im wesentlichen abgeschlossen ist, ergibt sich bei einem Temperaturgradienten von etwa 1000 Grad $\cdot \mathrm{cm}^{-1}$ für die Transportwärme der Thermodiffusion in Wolfram ein Wert von $Q^{*}=5,6 \mathrm{kcal}$.

Die Theorie der Thermodiffusion in Metallen ist in einer Reihe von Arbeiten ${ }^{9-16}$ ausführlich behandelt worden. Während die Transportwärme allgemein als Differenz der Bildungs- und Wanderungsenthalpie der Leerstellen nach der Beziehung

$$
Q^{*}=H_{\mathrm{B}}^{\mathrm{a}}-H_{\mathrm{W}}^{\square}
$$

angesetzt wird, kommt $\mathrm{W}_{\text {EvER }}{ }^{16}$ auf Grund kinetischer Überlegungen zu der Beziehung

$$
Q^{*}=G_{\mathrm{B}}^{\mathrm{a}}-\beta G_{\mathrm{W}}^{\mathrm{D}},
$$

die sich aus der Differenz der freien Enthalpien für die Bildung und Wanderung von Leerstellen ergibt. Die Wanderungsenthalpie ist dabei noch mit einem Faktor $\beta$ versehen, der zwischen den Werten +1 und -1 liegt und für einen Leerstellenmechanismus den Wert +1 besitzt. Der Unterschied der beiden Beziehungen liegt in der Berücksichtigung des Entropieanteils nach $G=H-T S$ in der Beziehung (4). Da die einzelnen Größen der Leerstellenbildung und -wanderung für Wolfram bekannt sind, läßt sich die Transportwärme leicht berechnen (Tab. 1).

Die Transportwärme für den Thermotransport von Wolfram ergibt sich danach aus der Bildungsund Wanderungsenthalpie der Leerstellen zu $Q^{*}=$ $34,5 \mathrm{kcal}$ bzw. 1,5 eV. Die Berücksichtigung des Entropieanteils ergibt unter Zugrundelegung der von Schultz gemessenen Bildungsentropie der Leerstellen einen Wert von $Q^{*}=37,7 \mathrm{kcal}$ bzw. 1,63 eV, der nicht viel von dem nach (3) erhaltenen Wert abweicht, während sich aus dem von Kraftmakher

\begin{tabular}{|c|c|c|c|c|c|c|c|}
\hline$H_{\mathrm{B}}^{\square}$ & $S_{\mathrm{B}}^{\square}$ & $H_{\mathrm{w}}^{\mathrm{a}}$ & \multicolumn{2}{|c|}{$S_{\mathrm{W}}^{\mathrm{a}}$} & \multicolumn{2}{|c|}{$Q^{*}=H_{\mathrm{B}}^{\square}-H_{\mathrm{W}}^{口}$} & \multicolumn{2}{|c|}{$Q^{*}=G_{\mathrm{B}}^{\text {口 }}-\beta G_{\mathrm{W}}^{\square}$} \\
\hline $\mathrm{kcal}$ & $\mathrm{cal}$ & $\mathrm{kcal}$ & $\mathrm{cal}$ & $\mathrm{kcal}$ & $\mathrm{eV}$ & $\mathrm{kcal}$ & $\mathrm{eV}$ \\
\hline $76,1^{18}$ & $2,78^{18}$ & $39,8^{17}$ & $3,50^{17}$ & 36,3 & 1,57 & 37,7 & 1,63 \\
$72,5^{19}$ & $12,9^{19}$ & & & 32,7 & 1,42 & 14,0 & 0,61 \\
\hline
\end{tabular}

Tab. 1. Bildungs- und Wanderungsenthalpie bzw. -entropie von Leerstellen für Wolfram sowie die daraus berechnete Transportwärme der Thermodiffusion (berechnet für $T=2000^{\circ} \mathrm{K}$ ).

8 R. L. Andelin, J. D. Knight u. M. Kahn, Trans. AIME 233, 19 [1965].

9 W. Shockley, Phys. Rev. 91, 1563 [1953] ; 93, 345 [1954].

10 J. A. Brinkmann, Phys. Rev. 93, 345 [1954].

11 A. D. LeClaire, Phys. Rev. 93, 344 [1954].

12 R. W. Keyes, Phys. Rev. 93, 1389 [1954].

13 A. R. Allnatt u. S. A. Rice, J. Chem. Phys. 33, 573 [1960].
14 R. A. Oriani, J. Chem. Phys. 34, 1773 [1961].

15 P. G. Shewmon, J. Chem. Phys. 29, 1032 [1958].

16 H. W WVER Z. Naturforschg. 18 a, 1215 [1963].

17 H. Schultz, Z. Naturforschg. 14 a, 361 [1959].

18 H. Schultz, Acta Met. 12, 761 [1964].

19 Ya. A. Kraftmakher u. P. G. Strelkov, Fiz. Tverd. Tela 4, 2271 [1962]. 
ermittelten Wert der Bildungsentropie eine Transportwärme von $Q^{*}=14,0 \mathrm{kcal}$ bzw. $0,61 \mathrm{eV}$ ergibt. Der Vergleich mit dem aus der Oberflächenstruktur abgeschätzten Wert $Q^{*}=5,6$ kcal bzw. $0,25 \mathrm{eV}$ läßt dabei den letzteren Wert wahrscheinlicher erscheinen. Dabei sei darauf hingewiesen, daß diese Werte nur einer Abschätzung, nicht aber einer direkten experimentellen Bestimmung entspringen.

\title{
IR-Untersuchungen heterocyclischer Verbindungen
}

I. Intensitätsmessungen der CH-Valenzschwingungen einiger unsubstituierter heterocyclischer Fünfringe des Typs $\mathrm{C}_{4} \mathrm{H}_{4} \mathrm{X}$ und deren Benzologen

\author{
R. Joeckle, E. Lemperle und R. Mecke
}

Institut für Elektrowerkstoffe der Fraunhofergesellschaft, Freiburg i. Br.

(Z. Naturforschg. 22 a, 395-402 [1967] ; eingegangen am 6. Dezember 1966)

Die CH-Intensitäten heterocyclischer Fünfringe sind bedeutend kleiner als die der entsprechenden homocyclischen Verbindungen. Die Intensitäten nehmen bei den einfachen Fünfringen $\left(\mathrm{C}_{4} \mathrm{H}_{4} \mathrm{X}\right)$ in der Reihenfolge $\mathrm{X}=\mathrm{S}, \mathrm{NH}, \mathrm{O}$ ab, bei deren Benzologen $\left(\mathrm{C}_{8} \mathrm{H}_{6} \mathrm{X}\right.$ und $\left.\mathrm{C}_{12} \mathrm{H}_{8} \mathrm{X}\right)$ in der Reihenfolge $\mathrm{NH}, \mathrm{S}, \mathrm{O}$. Die Intensitätsabnahme ist von einer Verschiebung des Bandenschwerpunktes der $\mathrm{CH}$-Valenzschwingungen ins Kurzwellige begleitet. Durch Einbau weiterer Hetero-N-Atome in den Ring werden die Effekte auf $I_{\mathrm{CH}}$ und $\bar{\nu}_{\mathrm{CH}}$ verstärkt. Es wurde versucht, die beobachteten Resultate sowohl durch Änderung des Hybridisierungsverhältnisses in den CH-Orbitalen als auch durch die unterschiedliche Elektronen-Saugwirkung der Heteroatome und die damit verbundene geänderte Ladungsverteilung im Molekül qualitativ zu erklären.

Durch systematische Intensitätsmessungen der aromatischen CH-Valenzschwingungen von Benzol und Benzolderivaten konnten Substituenteneffekte auf die CH-Bindung quantitativ erfaßt werden ${ }^{1-6}$. Darüber hinaus zeigte es sich, daß die integralen $\mathrm{CH}$ Intensitäten $\left(I_{\mathrm{CH}}\right)$ in diesen Verbindungen sich aus voneinander unabhängigen Beiträgen der einzelnen CH-Bindungen additiv zusammensetzen ${ }^{4,7}$. Im unsubstituierten Benzol ergibt sich als $\mathrm{CH}$-Bindungsintensität ein Wert von $I_{\mathrm{B}}=0,099 \mathrm{~cm}^{3} / \mathrm{Mol}^{7}$.

In diesem Zusammenhang erhob sich die Frage, welchen Einfluß Ring-Heteroatome auf die Gruppenintensitäten der $\mathrm{CH}_{\mathrm{ar}}$-Valenzschwingungen ausüben. Die Lösung dieses Problems wurde zunächst durch Messungen an Pyridin und dessen Derivaten versucht ${ }^{8-10}$. Dabei fand man für die CH-Intensität von Pyridin einen Wert von $I_{\mathrm{CH}, \mathrm{Py}}=0,48_{2}$. Nach

1 E. D. Schmid u. J. Bellanato, Z. Elektrochem. 65, 362 [1961].

2 E. D. Sснмір, Ber. Bunsenges. Physik. Chem. 67, 39 [1963].

3 E. D. Schmid, V. Hoffmans, R. Joeckle u. F. Langenbucher, Spectrochim. Acta 22, 1615 [1966].

4 E. D. Schmid u. F. Langenbucher, Spectrochim. Acta 22, 1621 [1966].

5 E. D. Schmid u. V. Hoffmann, Spectrochim. Acta 22, 1633 [1966].

6 E. D. Sснмid, Spectrochim. Acta 22, 1659 [1966].

7 F. Langenbucher u. R. Mecke, Spectrochim. Acta 21, 1287 [1965]. diesem Resultat sollte das N-Heteroatom keinen nachweisbaren Einfluß auf die CH-Bindungsintensitäten in Pyridin haben, da ein Wert von $0,49_{5}\left(5 \times I_{\mathrm{B}}\right)$ zu erwarten ist, wenn die CH-Bindungsintensität in Benzol auch für Pyridin gilt. Andererseits liegt die Vermutung nahe, daß die Übereinstimmung beider Werte auf einem numerischen Zufall beruht. Dafür sprechen die Ergebnisse, die man bei der Auswertung der einzelnen Banden des Pyridinspektrums erhält ${ }^{8}$, die sehr geringe Intensität von Pyridin-N-oxid ${ }^{11}$ sowie die CD-Intensitäten teilweise deuterierten Pyridins ${ }^{12}$.

Im Rahmen dieser Untersuchungen wird das Verhalten weiterer heterocyclischer Systeme, wie Thiophen, Pyrrol, Furan, deren Benzologe sowie Substanzen mit mehreren Heteroatomen studiert. Bei diesen Verbindungen ist der aromatische Charakter

8 E. D. Schmid u. R. Joeckle, Spectrochim. Acta 22, 1645 [1966].

9 R. Joeckle, E. D. Schmid u. R. Mecke, Z. Naturforschg. 21 a, 1906 [1966].

10 R. Joeckle u. R. Mecke, Ber. Bunsenges. Physik. Chem., im Druck.

11 Die CH-Intensität von Pyridin-N-oxid beträgt ca. $0,15 \mathrm{~cm}^{3}$ /Mol; sie ist noch erheblich kleiner als diejenige von $\mathrm{Ni}$ trosobenzol (ca. $0,25 \mathrm{~cm}^{3} / \mathrm{Mol}$ ). R. JoECKLE, unveröffentlichte Messungen.

12 Erste Messungen deuterierter Pyridinisotope zeigen unterschiedliche Bindungsintensitäten in den einzelnen Stellungen. E. D. SснміD, private Mitteilung. 